\title{
Co-registration of MOLA profiles to HRSC DTMs for mapping local seasonal ice cover height variations at the Martian poles
}

\author{
Haifeng Xiao ${ }^{1}$, Alexander Stark ${ }^{2}$, Gregor Steinbrügge ${ }^{3}$, Gregory Neumann ${ }^{4}$, and Jürgen Oberst ${ }^{1,2}$ \\ ${ }^{1}$ Technical University of Berlin, Institute of Geodesy and Geoinformation Science, Berlin, Germany (haifeng.xiao@campus.tu- \\ berlin.de) \\ ${ }^{2}$ Institute of Planetary Research, German Aerospace Center (DLR), Berlin, Germany \\ ${ }^{3}$ Department of Geophysics, Stanford University, Stanford, USA \\ ${ }^{4}$ Planetary Geodynamics Laboratory, NASA Goddard Space Flight Center, Greenbelt, USA
}

\begin{abstract}
We propose the co-registration of local laser profile segments to high resolution Digital Terrain Models (DTMs) as an approach for obtaining seasonal $\mathrm{CO}_{2}$ ice cover height variations on Mars. The co-registration is parameterized in instantaneous MOLA pointing angles involving a rigorous laser altimeter geolocation model. Thereby, the height bias of the MOLA footprint produced by the pointing bias could be compensated through an iterative process. The feasibility and advantages of this method are tested in an example region. The ultimate goal is to apply this method to Mars Orbiter Laser Altimeter (MOLA), SHAllow RADar (SHARAD) radar altimetry and high-resolution stereoscopic DTMs to generate long-term seasonal height change time series at the Martian poles with high spatial and temporal resolution.
\end{abstract}

\section{Introduction}

The dynamic growth and retreat of polar $\mathrm{CO}_{2}$ frost at the Martian poles has always been one of the focuses of planetary scientists $[1,2,3]$. Accurate measurements of seasonal and long-term elevation and volume changes can serve as important constraints in Mars climate models, help tap into the density evolution of the $\mathrm{CO}_{2}$ snow once combined with gravity measurements, and constrain the degree of stability of the polar deposits. The commonly-used approach to this problem is the cross-over analysis of the laser altimetry profiles, but this method may suffer from significant interpolation errors when spacing between footprints is large, also residual pointing, timing and orbit error may translate into lateral shifts of the laser profiles and further undermine the results. Here, we propose and validate the local co-registration between laser profile segments and high resolution DTMs from stereo pairs as a solution to these limitations.

\section{Data}

\subsection{MOLA records}

The MOLA Precision Experimental Data Record (PEDR) dataset features a total of 8505 
profiles, acquired in the mapping and extended phases of Mars Global Surveyor (MGS) from February, 1999 to May, 2001, which spanned approximately a full Martian year [1]. The PEDR dataset was processed using MGS orbit trajectory model and a Mars rotational model by GSFC dating back to 2003. Therefore, we have incorporated a refined orbit model from [4] and IAU2015 Mars rotational model [5] in the MOLA geolocation reprocessing. Meanwhile, to account for the special relativity effect, the pointing aberration correction has also been taken care of in the reprocessing [6].

\subsection{HRSC DTMs}

The High Resolution Stereo Camera (HRSC) is a pushbroom camera onboard the European Space Agency (ESA) spacecraft Mars Express. A total of 34 HRSC DTM tiles are adopted which feature a grid size of $50 \mathrm{~m}$ and covers the majority of the Martian South Pole [7].

\section{Methods}

First, the reprocessed MOLA profiles are self-registered to each other to form a coherent reference in the Martian South Pole [8, 9]. Subsequently, individual HRSC DTM tiles have been aligned to the MOLA reference data and mosaiced to a self-consistent reference for the co-registration. The coregistration of the reprocessed MOLA profiles to the aligned HRSC DTM mosaic is setup by compensating for two alignment angles of the boresight and height which incorporates an analytical laser altimeter geolocation model. The benefit is that the height offset induced by bias in pointing can be simultaneously compensated during the iterative co-registration process. The height differences with respect to the DTM mosaic at either footprint, cross-overs, or pseudo cross-overs are assigned as the height corrections from the local co-registration process using segmented profiles centered at these feature points. Here, pseudo cross-overs are DTM-based and formed by two track segments that do not have to actually intersect, substantially increasing the available number of cross-overs [10]. Then, $\mathrm{CO}_{2}$ height change time series are obtained by median-binning those temporal height differences with the uncertainty quantified by scaled median absolute deviation. To get rid of a temporal systematic error, the acquired temporal trend is subtracted from the one at $60^{\circ} \mathrm{S}$ annulus which features unchanging topography.

\section{Results}




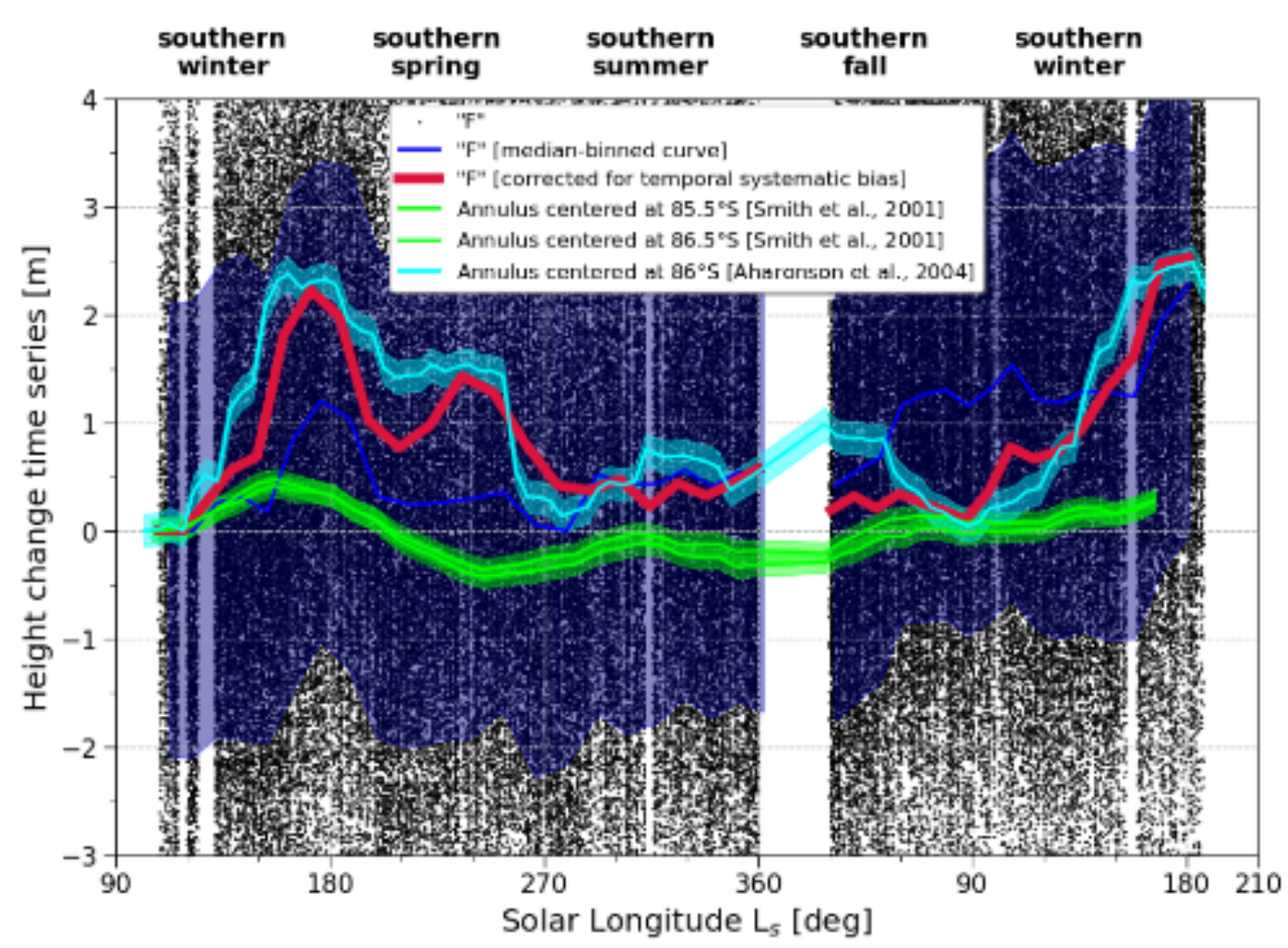

Figure 1: Results using height differences at footprints before the adoption of the local coregistration procedure ("F_LC", red line, bottom) and comparison to previous literature (lime and aqua lines).

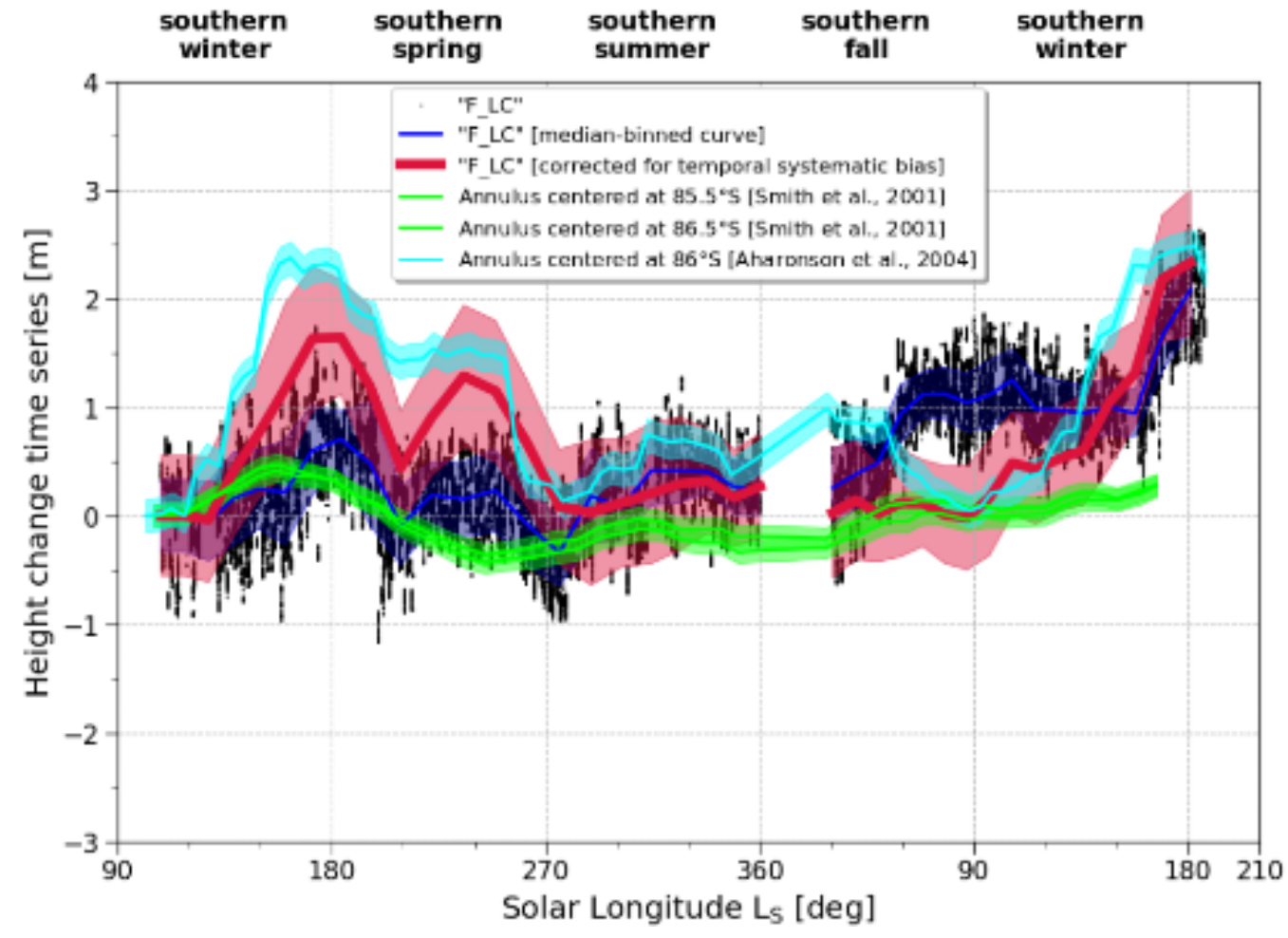

Figure 2: Results using height differences at footprints after the adoption of the local co-registration procedure ("F_LC", red line, bottom) and comparison to previous literature (lime and aqua lines). 
A test has been carried out at an example region situated right on the residual ice cap where largest peak-to-peak height variation would be expected. The uncertainty of the derived height change time series using height differences at footprints decreased from $\sim 2 \mathrm{~m}$ to $\sim 0.5 \mathrm{~m}$ after the adoption of the local co-registration procedure, roughly a fourfold enhancement (Figures 1 and 2). The maximum height fluctuation is estimated at $\sim 2.5 \mathrm{~m}$ which is similar to that of Aharonson et al. (2004) [2]. The suspicious off-season accumulation pointed out by previous results using MOLA has not been resolved. While a sharp pit-shaped feature of $\sim 1 \mathrm{~m}$ depth centered at solar longitude $210^{\circ}$ is observed. Similar results have been obtained using height differences at cross-overs and pseudo cross-overs. As a byproduct of the co-registration process, MOLA alignment angles have also been examined to follow some specific temporal patterns.

\section{Summary}

We show the feasibility and merits of the local co-registration strategy in the application of retrieving height changes at either footprints, cross-overs, or pseudo cross-overs. As for the next step, we will put the SHARAD radar altimetry and reflectometry into test [11]. Combined with the MOLA laser altimetry, long-term $\mathrm{CO}_{2}$ height change time series spanning two decades could possibly be retrieved. Apart from height change mapping, the proposed method could also be readily adapted to the tidal Love number measuring, orbit refinement with so called "direct altimetry" [12] and others.

\section{Acknowledgements}

This work was supported by a research grant from Helmholtz Association and German Aerospace Center (DLR). We acknowledge the work by the MOLA and HRSC instrument and science teams.

\section{References}

[1] Smith et al.,Science, 2001, 294, 2141-2146.

[2] Aharonson et al., JGRPlanets, 2004, 109(E5).

[3] Genova, ActaAstronaut., 2020, 166, 317-329.

[4] Konopliv et al., Icarus, 2006, 182, 23-50.

[5] Konopliv et al., Icarus, 2016, 274, 253-260.

[6] Xiao et al., Submitted to JoG.

[7] Putri et al., PSS, 2019, 174, 43-55.

[8] Stark et al., EPSC2018, Contrib. No. 890.

[9] Stark et al., GRL, 2015, 429, 7881-7889.

[10] Barker et al., Icarus, 2016, 273, 346-355.

[11] Steinbrügge et al., LPSC2019, LPI Contrib. No. 2132.

[12] Goossens et al., Icarus, 2020, 336, 113454. 